
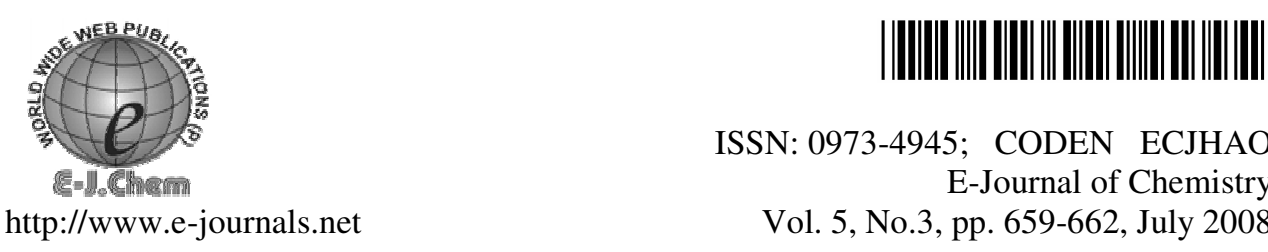

ISSN: 0973-4945; CODEN ECJHAO
E-Journal of Chemistry
Vol. 5, No.3, pp. 659-662, July 2008

NOTE

\title{
Schrodinger Equation for the Hydrogen Atom - A Simplified Treatment
}

\author{
L.R.GANESAN and M.BALAJI* \\ Former Professor of Chemistry, Madura College, Madurai. \\ *Department of Chemistry, Ramakrishna Mission Vivekananda College, Chennai. \\ balajimanisharma@gmail.com
}

Received 4 November 2007; Accepted 2 January 2008

\begin{abstract}
A simple method is presented here for solving the wave mechanical problem of the hydrogen atom. The normal method of converting the Cartesian coordinates into polar coordinates is tedious and also requires an understanding of the Legendre and Lagurre polynomials. In this paper we are using an alternative method, which requires only minimal familiarity with mathematical concepts and techniques.
\end{abstract}

Keywords: Schrödinger equation, Euler's theorem on homogeneous functions, Laplace equation.

The equation ${ }^{1} \nabla^{2} \psi+\left(\frac{2 \mu}{\hbar^{2}}\right)\left(\mathrm{E}+\frac{\mathrm{e}^{2}}{\mathrm{r}}\right) \psi=0$ is usually solved by expressing ' $\nabla^{2}$ ' in spherical polar coordinates $^{2-4}$. The following is an alternative treatment of the same problem. Implicitly it is equivalent to the usual textbook treatment but explicitly it is easier to follow

The following assumptions are made. Their justification is a posteriori, since they yield the correct solution.

\section{Assumption 1}

Since the potential energy contains $\mathrm{r}$ explicitly, we choose $\psi$, a product function i.e., $\psi=\psi_{1} \psi_{2}$ where $\psi_{1}$ depends on $\mathrm{r}$ alone, while no restriction of a similar kind is placed on $\psi_{2}$; we have thus, a certain freedom regarding the choice of $\psi_{2}$. We exercise this freedom in selecting a convenient form for $\psi_{2}$.

Thus

$$
\nabla^{2}\left(\psi_{1} \psi_{2}\right)+\left(\frac{2 \mu}{\hbar^{2}}\right)\left(\mathrm{E}+\frac{\mathrm{e}^{2}}{\mathrm{r}}\right)\left(\psi_{1} \psi_{2}\right)=0
$$




$$
\psi_{2}\left(\nabla^{2} \psi_{1}\right)+\psi_{1}\left(\nabla^{2} \psi_{2}\right)+2\left(\operatorname{grad} \psi_{1}\right) \cdot\left(\operatorname{grad} \psi_{2}\right)+\left(\frac{2 \mu}{\hbar^{2}}\right)\left(\mathrm{E}+\frac{\mathrm{e}^{2}}{\mathrm{r}}\right)\left(\psi_{1} \psi_{2}\right)=0
$$

Note: $\operatorname{grad} \psi_{1}=$ gradient vector of $\psi_{1}$

$$
=\overrightarrow{\mathrm{i}} \frac{\partial \psi_{1}}{\partial \mathrm{x}}+\overrightarrow{\mathrm{j}} \frac{\partial \psi_{1}}{\partial \mathrm{y}}+\overrightarrow{\mathrm{k}} \frac{\partial \psi_{1}}{\partial z}
$$

$\left(\operatorname{grad} \psi_{1}\right) .\left(\operatorname{grad} \psi_{2}\right)$ is the dot product of the gradient vectors i.e.,

$$
\frac{\partial \psi_{1}}{\partial \mathrm{x}} \frac{\partial \psi_{2}}{\partial \mathrm{x}}+\frac{\partial \psi_{1}}{\partial \mathrm{y}} \frac{\partial \psi_{2}}{\partial \mathrm{y}}+\frac{\partial \psi_{1}}{\partial \mathrm{z}} \frac{\partial \psi_{2}}{\partial \mathrm{z}}
$$

\section{Assumption 2}

We now choose $\Psi_{2}$ to be (i) a homogeneous function of degree $\ell$ in (x,y, z), of such a nature that (ii) it is a solution of the equation " $\nabla^{2} \Psi=0$ " (Laplace equation ${ }^{5}$ ), such a function is called a spherical harmonic. The advantages of this choice are two. First, the term $\nabla^{2} \Psi_{2}$ drops out in the equation (2) above. Further since $\psi_{1}$ is a function of $r$ alone,

$$
\frac{\partial \psi_{1}}{\partial \mathrm{x}}=\frac{\partial \psi_{1}}{\partial \mathrm{r}} \times \frac{\partial \mathrm{r}}{\partial \mathrm{x}}=\frac{\mathrm{x}}{\mathrm{r}} \frac{\partial \psi_{1}}{\partial \mathrm{r}}
$$

with similar expressions for $y$ and $z$. Therefore, the term

$$
2\left(\operatorname{grad} \psi_{1}\right) \cdot\left(\operatorname{grad} \psi_{2}\right)=\frac{2}{\mathrm{r}} \frac{\mathrm{d} \psi_{1}}{\mathrm{dr}}\left(\mathrm{x} \frac{\partial \psi_{2}}{\partial \mathrm{x}}+\mathrm{y} \frac{\partial \psi_{2}}{\partial \mathrm{y}}+\mathrm{z} \frac{\partial \psi_{2}}{\partial \mathrm{z}}\right)
$$

and this, by Euler's theorem ${ }^{6}$, becomes $\left(\frac{2 \ell \psi_{2}}{\mathrm{r}}\right) \frac{\mathrm{d} \psi_{1}}{\mathrm{dr}}$

After cancellation of $\psi_{2}$ equation (2) may now be written as

$$
\nabla^{2} \psi_{1}+\frac{2 \ell}{\mathrm{r}} \frac{\mathrm{d} \psi_{1}}{\mathrm{dr}}+\left(\frac{2 \mu}{\hbar^{2}}\right)\left(\mathrm{E}+\frac{\mathrm{e}^{2}}{\mathrm{r}}\right) \psi_{1}=0
$$

It is now easy to show that $\psi_{1}=\mathrm{e}^{-\mathrm{kr}}$ is a solution of equation (3a) viz

$$
\frac{\mathrm{d}^{2} \psi_{1}}{\mathrm{dr}^{2}}+\frac{2(\ell+1)}{\mathrm{r}} \frac{\mathrm{d} \psi_{1}}{\mathrm{dr}}+\left(\frac{2 \mu}{\hbar^{2}}\right)\left(\mathrm{E}+\frac{\mathrm{e}^{2}}{\mathrm{r}}\right) \psi_{1}=0
$$

Substitution of $\psi_{1}=e^{-k r}$ and simplification gives in this case

$$
\mathrm{K}^{2}-\frac{2(\ell+1)}{\mathrm{r}} \mathrm{K}+\left(\frac{2 \mu}{\hbar^{2}}\right)\left(\mathrm{E}+\frac{\mathrm{e}^{2}}{\mathrm{r}}\right)=0
$$

Equating separately to zero, terms involving $\frac{1}{r}$ and terms independent of $\mathrm{r}$, one gets

$$
\begin{aligned}
& \mathrm{E}=-\left(\frac{\hbar^{2}}{2 \mu}\right) \mathrm{K}^{2} \text { and }\left(\frac{\mu \mathrm{e}^{2}}{\hbar^{2}}\right)=(\ell+1) \mathrm{K} \\
& \therefore \mathrm{E}=-\left(\frac{2 \pi^{2} \mu \mathrm{e}^{4}}{(\ell+1)^{2} \mathrm{~h}^{2}}\right)
\end{aligned}
$$


It is further possible to select a function $\psi_{2}$ for each value of $\ell$.

\section{Note}

\begin{tabular}{cc}
\hline$\ell$ & $\psi$ \\
\hline 0 & Constant \\
1 & $\mathrm{x}, \mathrm{y}, \mathrm{z}$ \\
2 & $\mathrm{xy}, \mathrm{zx}, \mathrm{yz},\left(\mathrm{x}^{2}-\mathrm{y}^{2}\right)$ \\
.. & $\left(\mathrm{x}^{2}-\mathrm{z}^{2}\right),\left(\mathrm{y}^{2}-\mathrm{z}^{2}\right)$ \\
\hline
\end{tabular}

The six functions chosen for $\psi_{2}$ for $\ell=2$ are not, all linearly independent. Thus one chooses $x y, x z, y z,\left(x^{2}-y^{2}\right)$ and a linear combination of $\left(x^{2}-z^{2}\right)$ and $\left(y^{2}-z^{2}\right)$ as five linearly independent functions.

\section{Assumption 3}

The choice $\psi_{1}=\mathrm{e}^{-\mathrm{kr}}$ is too restrictive. One may therefore try $\psi_{1}=\mathrm{fe}^{-\mathrm{kr}}$, where $\mathrm{f}=\sum_{\mathrm{p}=0}^{\mathrm{t}} \mathrm{a}_{\mathrm{p}} \mathrm{r}^{\mathrm{p}}$ is a polynomial in $r$ of degree t. Equation (3) now gives.

$$
\begin{aligned}
& (D-K)^{2} f+\frac{2(\ell+1)}{r}(D-K) f+\left(\frac{2 \mu}{\hbar^{2}}\right)\left(E+\frac{e^{2}}{r}\right) f=0 \\
& \text { Where } D=\frac{d}{d r} .
\end{aligned}
$$

The coefficient of $r^{t}$ in this is $\left[K^{2}+\left(\frac{2 \mu E}{\hbar^{2}}\right)\right] a_{t}$.

Equating this to zero, we have recovered the relationship shown earlier between $\mathrm{E}$ and $\mathrm{K}$. Further the coefficient of $\mathrm{r}^{\mathrm{t}-1}$ is $\left[-2 \mathrm{Kt}-2(\ell+1) \mathrm{K}+\frac{2 \mu \mathrm{e}^{2}}{\hbar^{2}}\right] \mathrm{a}_{\mathrm{t}}$.

Equated to zero, this gives $\mathrm{K}=\left(\frac{\mu \mathrm{e}^{2}}{(\ell+\mathrm{t}+1) \hbar^{2}}\right)$

Thus $E=-\left(\frac{2 \pi^{2} \mu \mathrm{e}^{4}}{(\ell+\mathrm{t}+1)^{2} \mathrm{~h}^{2}}\right)$

We may now identify $(\ell+\mathrm{t}+1)$ with the principal quantum number, $\mathrm{n}$ and $\ell$ with the (so called) azimuthal quantum number. The choice " $\ell=0$ " gives the ' $s$ ' orbitals, for which $t=(n-1)$ is the degree of the polynomial ' $f$ ', for any given value of $n$. For $n=1,(\ell=0, t=0)$ is the only possible choice of $\ell$ and $\mathrm{t}, v i z$, the 1 s orbital. For $\mathrm{n}=2,(\ell=0, \mathrm{t}=1)$ gives the ' $2 \mathrm{~s}$ ' orbital, which is accidentally degenerate with $(\ell=1, \mathrm{t}=0)$ viz. the three $\mathrm{p}$ orbitals (see table 1 ).

Their functional forms are $\left(\mathrm{xe}^{-\mathrm{kr}}\right),\left(\mathrm{ye}^{-\mathrm{kr}}\right)$ and $\left(\mathrm{ze}^{-\mathrm{kr}}\right)$. With $\left.\mathrm{n}=3, \ell=0, \mathrm{t}=2\right),(\ell=1$, $\mathrm{t}=1)$ and $(\ell=2, \mathrm{t}=0)$ yield the $3 \mathrm{~s}, 3 \mathrm{p}$ and $3 \mathrm{~d}$ orbitals. The functional forms of the orbitals present no difficulty. An examination of equation (4) shows that the coefficient of $r^{\mathrm{s}}$ equated to zero gives

$$
\mathrm{a}_{\mathrm{s}+2}=\frac{-(\mathrm{t}-\mathrm{s}-1) 2 \mathrm{~K}}{(\mathrm{~s}+2)(2 \ell+\mathrm{s}+3)} \mathrm{a}_{\mathrm{s}+1}
$$


The largest and smallest values of $(\mathrm{s}+2)$ are $\mathrm{t}$ and 0 . Thus ' $\mathrm{s}$ ' varies from -2 to $(\mathrm{t}-2)$. The choice $\mathrm{s}=-2$ gives the result that $\mathrm{a}_{0}$ is indeterminate, since, in equation (5), $\mathrm{a}_{\mathrm{S}+1}$ and $(\mathrm{S}+2)$ are both zero fors $=-2$. For the other values of " $\mathrm{s}$ ", equation (5) is the recurrence relation for the coefficients of powers of ' $\mathrm{r}$ ' in $\mathrm{f}$.

e.g., 1

2 s orbital, $\ell=0, \mathrm{t}=1$,

$$
a_{S+2}=\left(\frac{S x 2 K}{(S+2)(S+3)}\right) a_{S+1} . \text { Put } S=-1 \mathrm{a}_{1}=-\left(\frac{2 \mathrm{~K}}{1 \times 2}\right) \mathrm{a}_{0} .
$$

Therefore $\mathrm{f}$ has the form $\mathrm{a}_{0}(1-\mathrm{kr})$ and $\psi$ has the form, (constant) $(\mathrm{I}-\mathrm{kr}) \mathrm{e}^{-\mathrm{kr}}$ e.g., 2

By a similar reasoning, we get the following results for $\mathrm{n}=3$

$$
\begin{gathered}
(\ell=0, \mathrm{t}=2): \quad \psi=(\text { constant })\left(1-2 \mathrm{kr}+\frac{2}{3} \mathrm{k}^{2} \mathrm{r}^{2}\right) \mathrm{e}^{-\mathrm{kr}} \\
(\ell=1, \mathrm{t}=1): \quad \psi=(\text { constant })\left(1-\frac{\mathrm{kr}}{2}\right) \mathrm{e}^{-\mathrm{kr}}(\mathrm{x} \text { or } \mathrm{y} \text { or } \mathrm{z}), \text { three functions. }
\end{gathered}
$$

$(\ell=2, \mathrm{t}=0): \psi=($ constant $) \mathrm{e}^{-\mathrm{kr}}$ (any of the five functions, $\mathrm{xz}, \mathrm{xy}, \ldots(c . f$ Table). The constants in the above forms may be determined by normalization. Thus, the method outlined above yields the real forms of the orbitals of the hydrogen like atom, in a straightforward manner. Its merit is that there is no need here to struggle with the Legendre and Lagurre differential equations.

The method gives the real forms of orbitals directly. This has the advantage of visualizing the shapes of orbitals, including their nodes, and overlaps in chemical bonding. Further one may easily construct their linear combinations as eigen functions of $\mathrm{L}_{\mathrm{z}}$ operator, if and when needed. For students and with no familiarity with the special functions of mathematical physics, the above method is mathematically transparent and demands no further knowledge than elementary calculus.

\section{References}

1. Schrödinger E, Ann.Physik, 1926, 79, 361.

2. Schrödinger E, Ann.Physik, 1926, 80, 437.

3. Epstein P S, Phys.Rev. 1926, 28, 695.

4. Walter I, Z.Physik, 1926, 38, 635.

5. Séroul R, Programming for Mathematicians, Springer-Verlag, 2000, p.15.

6. Shanks D, Solved and Unsolved Problems in Number Theory, $4^{\text {th }}$ Ed., 1993, p.21, 23-25. 


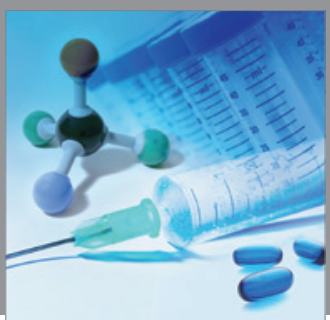

International Journal of

Medicinal Chemistry

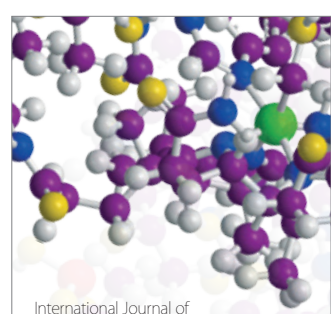

Carbohydrate Chemistry

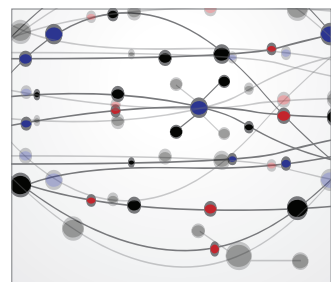

The Scientific World Journal
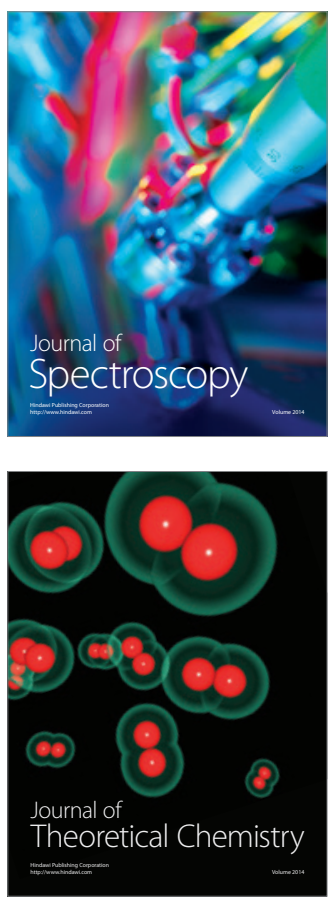
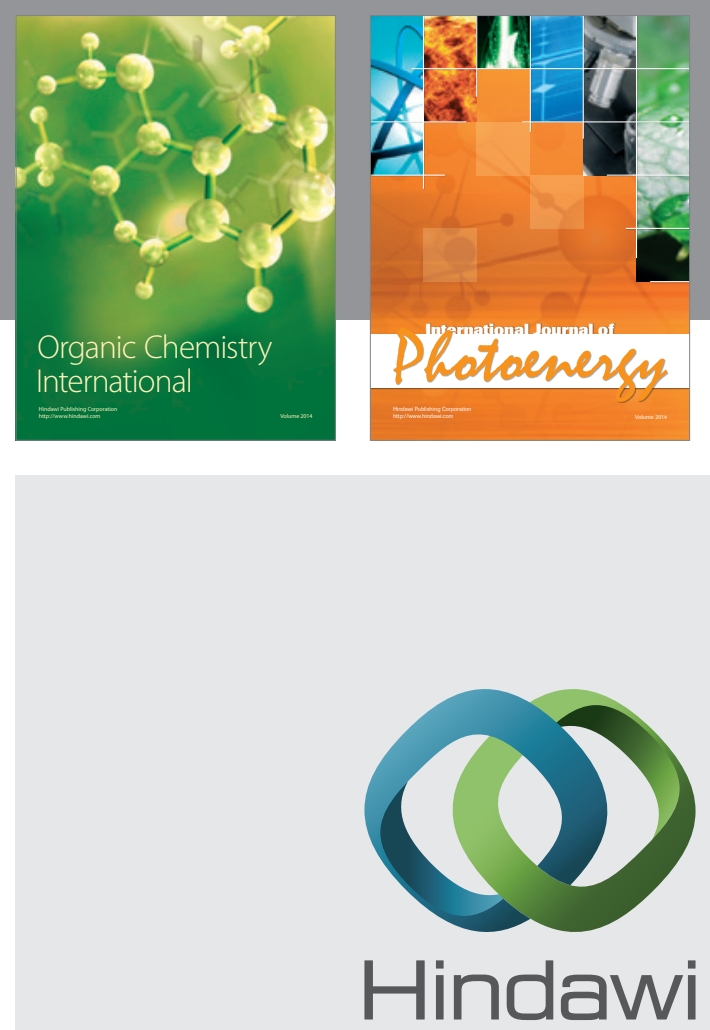

Submit your manuscripts at

http://www.hindawi.com
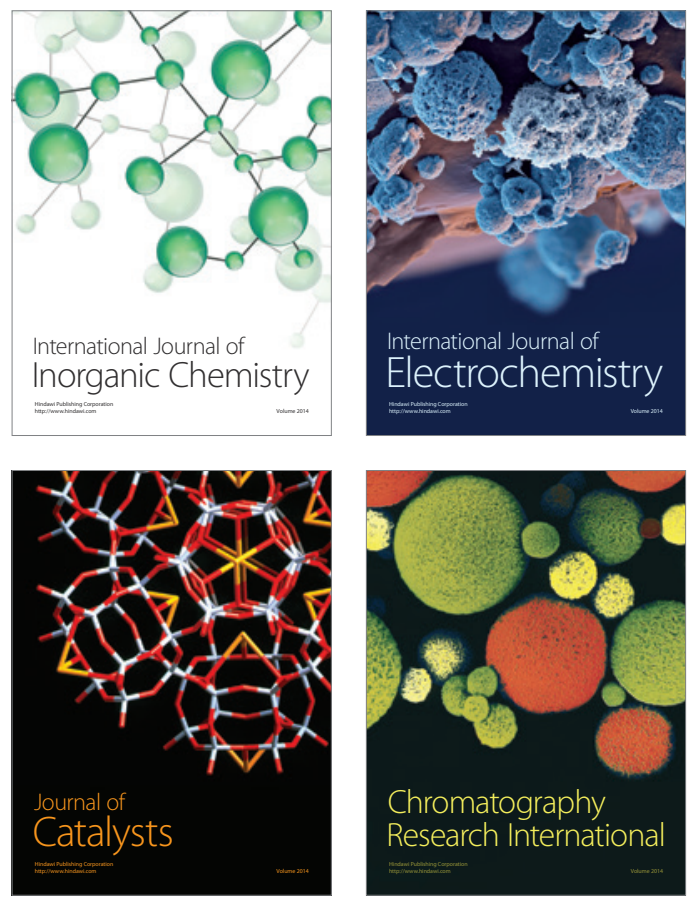
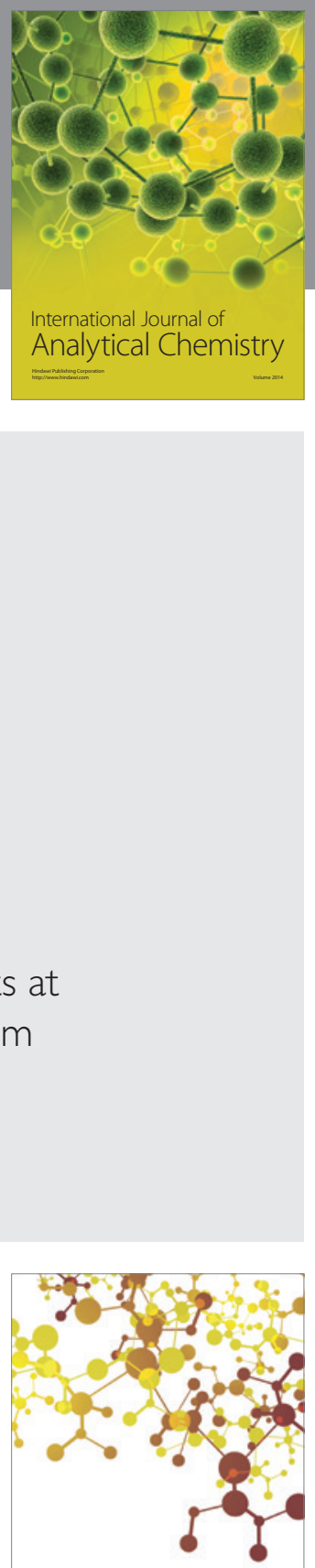

Journal of

Applied Chemistry
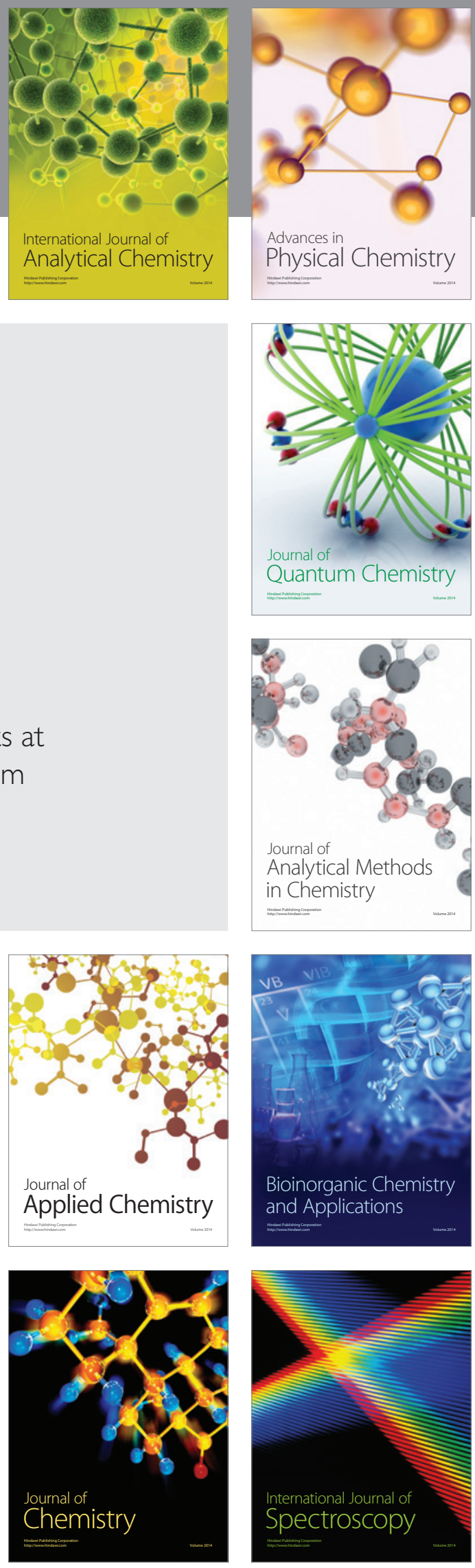